(Aus dem pathologisch-anatomischen Institut des Friedrichstädter Krankenhauses in Dresden.)

\title{
Beitrag zur pathologischen Histologie der gonorrhoischen Epididymitis.
}

Von

Dr. Johannes Wolf.

Mit 3 Textabbildungen.

Unter den entzündlichen Prozessen im Nebenhoden kommt neben der Epididymitis tuberculosa als die häufigste und klinisch wichtigste Erkrankung die gonorrhoische Epididymitis in Frage. Sie ist.eine fortgeleitete Entzündung im Gegensatz zu den bei septischen Erkrankungen, Pocken und anderen Infektionskrankheiten auftretenden Epididymitiden, die als hämatogen entstanden anzusprechen sind. Pathologischhistologisch sind bei beiden Prozessen die Bilder sehr ähnlich.

Außer den gonorrhoischen Epididymitiden treten fortgeleitete Entzündungen des Nebenhodens durch Infektionen mit anderen Mikroorganismen auf, so z. B. dem Tuberkelbacillus, Staphylococcus, Micrococcus catarrhalis und Bacterium coli. Müller erwähnt einen Fall von Coli-Epididymitis bei einem Individuum, das längere Zeit an einer Colipyelitis gelitten hatte. Bei der plötzlich auftretenden Epididymitis, die mit Vereiterung einherging, wurden bakteriologisch Colibacillen nachgewiesen. Reynolds lenkt die Aufmerksamkeit darauf, daß manche Fälle von gonorrhoischer Epididymitis durch die Anwesenheit von Colibacillen einen protrahierten Verlauf nehmen können. Ein Fall von Epididymitis, der hervorgerufen wurde durch den Diplococeus crassus, beschreiben Königsfeld und Salzmann. Dieser Diplococcus crassus besitzt die Semmelform und die Größe des Gonococcus Neisser, zeigt aber sowohl gram-positive als auch gram-negative Exemplare. Durch sehr exakt ausgeführte Untersuchungen stellten die Verfasser fest, daß dieser Diplococcus sich bakteriologisch und kulturell von den Gonokokken unterschied.

Alle dies durch Staphylokokken, Bacterium coli, Mikrokokken usw. hervorgerufenen Epididymitiden sind aber außer der Tuberkulose nicht so wichtig wie die durch den Gonococcus Neisser verursachten. Daß diese Entzündungen des Nebenhodens und des Samenleiters, die infolge einer gonorrhoischen Urethritis auftreten, durch den Gonokokkus selbst 
hervorgerufen werden, ist erst in den neunziger Jahren entdeckt und sichergestellt worden. Die Hypothese, daß die Epididymitis bei einer bestehenden gonorrhoischen Urethritis lediglich durch die Toxine der Gonokokken hervorgerufen würden, ist durch die späteren Untersuchungen und Befunde völlig widerlegt worden. Französische Autoren beschrieben einen besonderen Diplokokkus, den „Orchiococcus urethrae", als den Erreger der Epididymitis, die bei Bestehen der Harnröhrengonorrhoe auftritt. Dieser Orchiokokkus ist dem Gonokokkus sehr ähnlich, unterscheidet sich nur durch sein üppiges Wachstum auf verschiedenen serumfreien Nährböden und durch die stärkere Weißfärbung seiner Kolonien. Es scheint sehr wahrscheinlich, daß dieser Orchiokokkus identisch ist mit dem Diplococcus crassus von Königfeld und Salzmann. Es ist also nicht unwahrscheinlich, daß der Orchiokokkus eine Epididymitis hervorrufen kann, aber die Ansicht, daß er der Erreger der gonorrhoischen Epididymitis sei, ist energisch von der Hand $\mathrm{zu}$ weisen.

Es ist sehr einleuchtend, daß man seit langem bestrebt war, den Nachweis des wirklichen Erregers der gonorrhoischen Epididymitis bei der großen klinischen Bedeutung dieser Frkrankung zu erbringen. 1896 sprachen Neisser und Schäffer schon die nächstliegende und wahrscheinlichste Vermutung aus, daß nur der Gonokokkus selbst als der Erreger in Frage komme. Es gelang ihm jedoch nicht bei drei Fällen, trotz sorgfältigster wochenlang fortgesetzter Untersuchung einen positiven Gonokokkusnachweis zu erbringen. Aber trotzdem halten sie ihre Ansicht aufrecht, daß die Epididymitis bei Gonorrhoe eine durch den Gonokokkus hervorgerufene Erkrankung sei und daß der Fortpflanzungsweg der Entzündung von der Urethra durch den Ductus ejaculatorius und das Vas deferens geht, analog den Adnexerkrankungen bei der Gonorrhoe des Weibes. Im Jahre 1895 hatte Routier einen Fall von gonorrhoischer Epididymitis mit Vereiterung veröffentlicht. Er hatte in einem sich hinzugesellenden Absceß Gonokokken 'nachgewiesen. Jedoch finden sich keine Angaben, ob der Nachweis der Gonokokken in einwandfreier Weise geführt worden ist. Auch ein von Palmer berichteter Fall von gonorrhoischer Epididymitis mit positivem Gonokokkenbefund entbehrt näherer Angabe des Gonokokkennachweises.

Der erste sicher einwandfreie Nachweis, daß bei Bestehen der Gonorrhoe eine akut auftretende Epididymitis durch den Gonokokkus hervorgerufen wird, wurde 1897 von Groß und kurz darauf von Collan erbracht. Beide wiesen sowohl im Fiter des Exsudates als auch in den Kulturen den Gonokokkus nach. Diese Entdeckung wurde daraufhin bestätigt durch zahlreiche Mitteilungen in der Fachliteratur. Witte wies in einem Fall sicher Gonokokken nach. Andere Mikroorganismen 
fand er nicht. Sprecher beschreibt einen Fall von Gonorrhoe, die mit eiternder Prostatitis und Epididymitis kompliziert war und konnte nachweisen, daß die Nebenerkrankungen direkt oder ausschließlich von Neissers Gonokokkus abhängig waren, der sich als mit einer erheblichen Lebensfähigkeit und hohen Virulenz begabt zeigte. Ähnliche Befunde wurden noch von vielen anderen Autoren erhoben. Baer mann veröffentlichte 1903 die Ergebnisse seiner systematischen Untersuchungen an einem größeren Krankenmaterial und kommt zu dem Schluß, daß jede Epididymitis, die im Verlaufe einer gonorrhoischen Urethritis auftritt, durch lokale Invasion von Gonokokken bedingt ist. Andere wiederum, so Nobl und Sellei, konnten einen positiven Gonokokkenbefund nicht erbringen.

Trotzdem kann man heute wohl die Tatsache, daß lediglich der Gonokokkus der Erreger der Epididymitis bei Gonorrhoe ist, als gesichert annehmen. Wie erfolgt nun die Uberwandrung der Gonokokken von der Urethra nach dem Nebenhoden? Hor o vit z vertritt die lymphogene Infektion und nimmt an, daß das den Samenleiter begleitende Lymphgefäß als Propagationsbahn des gonorrhoischen Virus in Frage kommt. Ebenso vertreten Andry und Dalous den Standpunkt, daß die entzündliche Infiltration in Beziehung zu den Lymphgefäßen steht und bezeichnen die gonorrhoische Epididymitis als eine phlegmonöse Lymphangitis. Gegen diese lymphogene Propagation des Virus sprechen sich Nobl, Sellei und Simmonds aus. Oppenheim und Löw kamen an der Hand von neun Fällen und durch sehr exakt ausgeführte und zum größten Teil gelungene Versuche mit Kaninchen zu dem Schluß, daß die Momente, die als Ursache einer Epididymitis bei bestehender Urethritis posterior gonorrhoica anzusprechen sind, eine antiperistaltische Bewegung des Samenleiters hervorrufen können und daß auch durch diese retroperistaltische Bewegung das Virus tatsächlich vom Colliculus seminalis zu dem Nebenhoden gebracht wird. Veranlaßt zu den Untersuchungen wurden sie durch die Unistände und die oft blitzartige Entstehung der Epididymitis nach der Einwirkung des äthiologischen Momentes, z. B. eines Traumas, einer irritierenden Einspritzung usw. und das Auftreten der ersten Krankheitserscheinungen im Nebenhoden unter thoerspringen des Ductus deferens.

Nobl spricht auf Grund von Versuchen die Ansicht aus, daß nur der.Weg über den Ductus deferens für die Infektion von Bedeutung sein kann. Bei den blitzartig auftretenden Epididymitiden hält er es für wahrscheinlich, daß eben schon Gonokokken im Nebenhoden waren, aber ohne irgendwelche klinische Erscheinung zu machen. Erst das Trauma war das auslösende Moment. Nobl nimmt ein rasenförmiges Fortschreiten der Gonokokken auf der Schleimhaut der Samenwege an und begründet seine Annahme mit der normalen histologischen 
Beschaffenheit des Ductus deferens und des Ductus epididymidis. Tatsächlich stellte er auch in $60 \%$ der Fälle klinisch eine Veränderung des Vas deferens fest, bevor irgendwelche krankhaften Veränderungen am Nebenhoden auftraten.

Tritt nun eine Epididymitis plötzlich auf, obne daß vorher irgendwelche klinischen Erscheinungen von seiten des Ductus deferens wahrnehmbar wurden, muß man das rein mechanische Moment der rückläufigen Peristaltik des Samenleiters in Betracht ziehen, wenn man ein rasenförmiges Fortwachsen der Gonokokken auf der Schleimhaut ausschließen zu müssen glaubt, wegen des allzuraschen Auftretens der Epididymitis nach der gonorrhoischen Infektion. Diese antiperistaltige Bewegung des Ductus deferens bestätigt Schindler durch Experimente und läßt nur bei langdauernder Gonorrhoe und bei länger bestehender Urethritis posterior das rasenförmige Wachsen der Gonokokken gelten.

Über die Häufigkeit der Epididymitis gonorrhoica bei Gonorrhoe der Harnwege möchte ich einen Durchschnitt der Zahlen, die Jordan in seiner Arbeit gibt, anführen. Für poliklinische und Privatverhältnisse erhält man die Durchschnittszahl 11,0\%, für Krankenhausverhältnisse die Zahl 29,1\%. Es ist naturgemäß, daß diese Zahlen so verschieden sind.

Bevor ich die pathologischen Veränderungen bei der Epididymitis gonorrhoica bespreche, will ich erst einige Bemerkungen über die normale Histologie vorausschicken. Aus dem oberen Ende des Rete testis entwickeln sich die Ductuli efferentes testis, die, an Zahl etwa 10-i5, sich zu dem Ductus epididymidis vereinigen. Diese Ductuli efferentes bilden den Kopf des Nebenhodens. Der Ductus epididymidis ist stark geschlängelt und vielfach gewunden und bildet den Körper und den Schwanz des Nebenhodens. Aus dem Schwanz entwickelt sich der Ductus deferens, der scharf umbiegend, zuerst noch mäßig geschlängelt, dann gestreckt nach oben verläuft und zusammen mit den Nerven und Gefäßen als Funiculus spermaticus durch den Leistenkanal in das Becken tritt.

Der Ductus epididymidis besitzt ein zweireihiges Epithel, das aus inneren langen Cylinderzellen und äußeren basalen kubischen Zellen besteht. Die Cylinderzellen tragen an ihrem freien Ende zu Büscheln verklebte Flimmerhaare, die in gehärteten Präparaten als Kegel in das Kanälchenlumen hineinragen. Es folgt nach außen eine zarte Membrana propria, ein dünnes Geflecht elastischer Fasern und eine Schicht circulär verlaufender lockerer Muskelfasern. Alle diese Schichten sind von einem lockeren zell- und gefäßreichen Bindegewebe umgeben. Der Ductus deferens besitzt eine in Längsfalten gelegte Schleimhaut. Das Epithel gleicht im Anfange dem des Ductus epididymidis, dann wird es zu einem mehrreihigen Epithel. Die Basalzellen verschwinden und das 
Cylinderepithel wird dem Übergangsepithel sehr ähnlich. Die Flimmerhaare gehen verloren. Dem Epithel folgt eine mit einem dichten Geflecht elastischer Fasern durchflochtene Submucosa, an die sich nach außen zwei Lagen einer kräftigen Längsmuskulatur, die eine Ringmuiskellage in sich einschließen, anschließen. Diese drei Muskellagen sind ca. $1 \mathrm{~mm}$ dick und relativ gefäßarm.

Mit der Anatomie der gonorrhoischen Epididymitis beschäftigten sich verschiedene ältere Autoren wie Monteggia, Gausail, Scheperlern, Godard und einige andere. Die meisten Autoren befaßten sich nur mit den grobanatomischen Verhältnissen. Scheperlern ist wohl der erste, der eine mikroskopische Untersuchung der Epididymitis gonorrhoica beschrieb. Er fand die Wandungen des Nebenhodenkanals völlig zerstört durch eine aus Lymphocyten bestehende Infiltration. Das umgebende Gewebe war mit Eiterzellen durchsetzt. Godard fand neben der Epididymitis gonorrhoica eine Spermatocystitis. Andry und Dalous beschrieben eine Epididymitis, die mit Absceßbildung einherging, daß die Affektion von dem intertubulären Bindegewebe und von der Umgebung der Lymphgefäße ausging. Das Epithel erfuhr Metaplasie. Besonders wandelte sich das cylindrische Epithel des Ductus deferens zu einem glatten würfelförmigen Epithel um. Der Inhalt des Vas deferens bestand aus Leukocyten und Spermatozoen.

Die erste genauere Beschreibung der histologischen Veränderungen bei Epididymitis gonorrhoica stammt aus der Feder Nobls im Jahre 1903. Einer seiner Patienten, dessen Epididymitis sich bereits im Involutionsstadium befand, kam plötzlich an einer eingeklemmten Hernie zum Exitus, wodurch er Gelegenheit fand, den Hoden zu untersuchen. Im Samenstrang blieben die pathologischen Veränderungen auf den Ductus deferens beschränkt, während die weiteren musculären. (M. cremaster int.) und bindegewebjgen Umgebungsschichten fast als normal zu bezeichnen wären. Besonders die Intima ist befallen. Das Epithel hat die regelmäßige Anordnung verloren und ist z. T. in hochgradige Wucherungen übergegangen. Es haben sich Anzeichen von Desquamation und Proliferation geltend gemacht. Gleichzeitig sind Leukocyten und Lymphocyten in das Epithel eingewandert. Die Tunika propria ist durch Quellung und Vermehrung der Bindegewebsfibrillen und -kerne, Rundzelleneinlagerung und adventitielle Infiltration von Capillargefäßen erheblich verbreitert. Die entzündliche Lymphocyteninfiltration ist am dichtesten in einer schmalen subepithelialen Zone und verliert sich allmählich in der Innen- und Mittellage der Muskulatur. Alle diese Veränderungen nehmen zu in dem dem Nebenhoden näher gelegenen Abschnitte des Ductus deferens, in dem es durch die Epithelwucherung und die Infiltration der Submucosa zu einer Einengung des Lumens kommt. Im Nebenhoden sitzen die hauptsächlichsten 
pathologischen Veränderungen in der Cauda, während die Entzündungserscheinungen nach dem Kopf zu nachlassen. Im Bereich der Cauda zeigt der Nebenhodenkanal in der Mucosa die stärksten Veränderungen. Einzelne der Schlingen sind noch von dem hohen Cylinderepithel ausgekleidet und mit der circulären Muscularis scharf gegen die Umgebung abgesetzt; aber in den meisten Schlingen ist es zu einer Wucherung, Desquamation und Rundzelleninfiltration des Epithels gekommen. Die Tunica propria ist aufgelockert und infiltriert und die Muskelschicht ist verbreitert. Nach dem Kanälchenlumen zu liegen auf den Epithelien locker gefügte, gequollene Formen von Epithelzellen. In der Kanallichtung liegen isolierte abgestoßene Epithelzellen und Eiterkörperchen. In der Tunica propria sind dichte Mengen von einkernigen Rundzellen und vereinzelte polynucleäre Leukocyten. Diese Infiltrate dringen aber auch in die Epithelschicht und die Muscularis vor. Die Muscularis ist verbreitert und weist die erwähnten Infiltrationsherde auf, die auch noch in das weitere, den Nebenhoden umgreifende Bindegewebe, vordringen. Alle diese schwersten Formen der Entzündung wechseln $a b$ mit weniger schweren und ganz leichten, die dicht nebeneinander liegen können. Zu einer Einschmelzung der Infiltrate oder zu einer Kompression und Verlagerung der Kanälchen ist es nicht gekommen. In seinen Präparaten hat Nobl keine Gonokokken gefunden.

1904 erschien von Sellei eine Arbeit über Histologie der Epididymitis gonorrhoica. Er hat 15 Fälle, davon zwei akute, die übrigen inveterierte Epididymitiden untersucht. Er kommt zu dem Ergebnis, daß die Entzündung im intercanaliculären Gewebe destruktiv wirkt, während sich im Bindegewebe um die Kanälchen neue Bindegewebszellen bilden. Das Epithel der Ductuli efferentes ist teils schleimig degeneriert, teils losgelöst. Die Kanälchen sind stellenweise ausgedehnt, ja sogar soweit ausgedehnt, daß benachbarte zusammengeflossen sind. Im Epithel und im Gewebe darunter sind nur geringe Lymphocyteninfiltrationen zu sehen. Ebenso sind auch im Bindegewebe nur wenige Lymphocyten, fast keine Leukocyten, dagegen Plasmazellen und Fibroblasten in großen Mengen. Die Hauptveränderungen sind in den Kanälchen, besonders der Cauda. Im Caput epididymidis fand nur eine durch Bindegewebsvermehrung verursachte Zusammendrückung der Ductuli efferentes statt. Je ausgeprägter die Hyperplasie des Bindegewebes war, umso deutlicher trat neben der intracanaliculären Deformation eine Atrophie des canaliculären Systems des Nebenhodens auf. Bei einem der akuten Fälle war auch der Hoden in Mitleidenschaft gezogen. Auch Sellei konnte Gonokokken nicht nachweisen.

Des genaueren hat sich dann 1905 Baermann mit der Histologie der gonorrhoischen Epididymitis beschäftigt. Bei der akuten (am 3. Tage) fand er die erheblich erweiterten Kanälchen mit polynukleären Leuko- 
cyten vollgepfropft. Das Epithel ist gewuchert, am freien Rande zerfasert, und die Epithelzellen sind z.T. blasig aufgetrieben. Durch dieses Epithel wandern nun reichlichst Eiterkörperchen ins Lumen des Kanälchens hinein. An manchen Stellen ist das Epithel von der Basalmembran völlig abgelöst und durch die nachdrängenden Leukocyten in das Lumen vorgeschoben. In den im Lumen liegenden Leukocyten sind zahlreiche Gonokokken nachzuweisen. Wo das Epithel von der Basalmembran abgelöst ist, sieht man die Gonokokken in das umgebende Bindegewebe eintreten. Die Weiterverschleppung in das bindegewebige Zwischengewebe wird durch die Lymphbahn vermittelt. Wo das Epithel zugrunde gegangen ist, schiebt sich ein dichtes Infiltrat längs der Kapillen in die Ringmuskelschicht. Diese Infiltrate bestehen größtenteils aus polynucleären Leukocyten und zahIreichen Mastzellen. Zwischen zwei Kanälchen kann es zur Einschmelzung des Zwischengewebes und zum Durchbruch kommen. Schmelzen noch mehr Kanälchen ein, so kommt es schon zur Bildung kleiner Abscesse. Bei ganz foudroyanten Fällen kann das Nebenhodengewebe vollständig zerstört werden, so daß ein Kanälchen nur noch einen mit Blut und Eiter gefüllten Schlauch darstellt, in dem sich aber auch noch Gonokokken nachweisen lassen.

Bei der chronischen Epididymitis ist der distal von der erkrankten Stelle erweiterte Ductus deferens mit Spermatozoen angefüllt. Das Epithel ist teils abgeplattet, teils niedriger als normal. Eiterzellen sind gewöhnlich nicht zu finden. Dais Bindegewebe ist völlig normal. Die kranke Stelle zeigt an einzelnen Stellen einen Verschluß der ihres Epithels verlustig gegangenen Kanalschlingen. Das Lumen ist mit Granulationsgewebe und Mastzellen ausgefüllt; ebenso sind im umgebenden Bindegewebe einzelne Haufen von Granulationsgewebe. Im Lumen der nicht zerstörten Kanalschlingen finden sich zahlreiche polynucleäre Leukocyten und Mastzellen. Im gewucherten Epithel enthalten die blasigen Hohlräume ebenfalls zahlreiche Eiterzellen. Die zugrunde gegangenen Kanalschlingen sind meist, abgesehen von der Form des Infiltrates, an der feinen elastischen Faserung, die relativ wenig alteriert ist, gut zu erkennen. Gonokokken konnte Baerman $n$ bei den chronischen Fällen der Epididymitis nicht mehr nachweisen. Bei völlig abgelaufener Epididymitis tritt an Stelle des Granulationsgewebes ein junges zellreiches Bîndegewebe. Bei chronischer Defèrentitis sieht man, daß die Mucosa und das Epithel durch junges Bindegewebe ersetzt ist. Muscularis und die Elastica sind erhalten und halten eben den entzündungserregenden Einfluß der Gonokokken von den gefäßreichen Hüllen des Samenstranges ab.

Noch einige Arbeiten möchte ich nicht unerwähnt lassen. Dind und Métra u $x$ fanden auch in der Mucosa und Submucosa des Vas deferens reichliche zellige Infiltration, während die Muscularis freiblieb. 
Das Lumen enthielt Detritus von Leukocyten und desquamierten Epithelien. Delbet und Chevassu stellen fest, daß die starke Proliferation des canaliculären Epithels häufiger Stenosen und Verschluß des Kanälchens bedingen als die Wucherung im intercanaliculären Bindegewebe. In den Hodenkanälchen fanden sich nie Entzündungserscheinungen.

Bevor ich zur Besprechung des eignen Falles komme, möchte ich noch kurz den Ausgang der Epididymitis gonorrhoica erwähnen. Bei Eintreten der Regeneration bleiben die Infiltrate im Bindegewebe noch längere Zeit bestehen und können evtl. bei der chronischen Epididymitis zum Ausgangspunkt von Rezidiven werden. Sehr selten tritt eine Mitbeteiligung des Hodens ein. Als heikelste Folge ist wohl die Sterilität oder Azoospermie im Anschluß an eine Epididymitis gonorrhoica in Betracht zu ziehen. Entsteht im Verlauf der Erkrankung ein narbiger Verschluß der Kanalschlingen des Ductus epididymidis oder des Ductus deferens, so muß Azoospermie auf der erkrankten Seite auftreten. De Sinéty, Simmonds usw. sprechen sich des näheren über die Azoospermie als Folge der gonorrhoischen Epididymitis aus. Sohließlich möchte ich einen von Delbet veröffentlichten interessanten Fall nicht unerwähnt lassen. 11 Jahre nach einer Epididymitis wurde operativ eine Anastomose zwischen Nebenhoden und Vas deferens hergestellt. Ein Jahr nach der Operation traten nach jahrelanger Sterilität wieder Spermatozoen auf und veranlaßten eine Schwangerschaft.

Den bis jetzt beschriebenen Fällen von Epididymitis gonorrhoica kann ich einen neuen hinzufügen. Es handelt sich um einen operativ entfernten rechten Hoden. Vorausschicken muß ich, daß die klinische Diagnose sehr unsicher gestellt war; ich bringe deshalb einen Auszug aus der Krankengeschichte.

Es handelt sich um einen 51jährigen Patienten P. J.

Anamnese: den 27. I. 1919.

Pat. gibt an, daß er sich vor 3 Wochen beim Übersteigen eines Geländers an die Geschlechtsteile gestoßen und seit diesem. Tage Schmerzen am Hoden habe. Da der Hoden stark angeschwollen war, begab er sich zu einem Arzt in Behandlung, der Umschläge verordnet habe. Die Schwellung und Schmerzhaftigkeit nahmen aber ständig zu. Infectio ven. wird negiert. Ausfluß aus der Harnröhre bestand angeblich nie. Wegen Zunahme der Erscheinungen und Fiebers wurde Pat. einer hiesigen chirurgischen Privatklinik überwiesen und zunächst ambulant und abwartend behandelt und dann wegen weiterer Zunahme aller Symptome am 27. I. 1919 in die Klinik aufgenommen.

Status: 27. I. 1919.

Kräftiger Mann in gutem Ernährungszustand. Herz, Lungen und Nervensystem o. B. Eiweiß und Zucker negativ. Rechter Testikel stark verdickt; Scrotum geschwollen, entzündlich gerötet.

Diagnose 27. I. 1919. Entzündeter rechtsseitiger Hoden.

Am gleichen Tage wurde die Exstirpation des erkrankten Hodens ausgeführt, da man eine spezifisch-gonorrhoische Erkrankung ausschließen zu können glaubte, vielmehr mit größerer Wahrscheinlichkeit auf eine Tuberkulose des Hodens und 
des Nebenhodens schloß. Am folgenden Tage trat eitriger Ausfluß aus der Harnröhre auf, der nach einigen Tagen verschwand, um bald wieder aufzutreten. Erst nach einigen Einspritzungen durch den zuerst behandelnden Arzt (welcher Art konnte ich nicht erfahren) blieb der Ausfluß weg. Da der Patient trotz Aufforderungen sich nicht wieder beim Arzte vorstellte, kann ich keine weiteren Angaben über den Heilungsverlauf machen. Zum Schluß will ich noch bemerken, daß ich nachträglich feststellen konnte, daß der erstbehandelnde Arzt gleich zu Beginn der Erkrankung einen'geringen Ausfluß aus der Urethra festgestellt hat, aber ohne bakterioskopische Untersuchungen anzustellen.

Der exstirpierte Hoden wurde dem pathologisch-anatomischen Institut Dresden-Friedrichstadt zur Untersuchung zugeschickt und mir von Herrn Geheimrat Prof. Dr. Schmorl zur histologischen Untersuchung und Beschreibung überlassen.

Das Präparat, das in Formalin gehärtet ist, ist der aus dem Scrotum herausgeschälte Hoden mit Nebenhoden und kurzes Stück des Samenstranges mit Ductus deferens, Gefäßen und Nerven.

Makroskopisch sieht man am Hoden keinerlei Veränderungen. Dagegen ist die Cauda epididymidis hochgradig, das Corpus epididymidis in geringerm Maße, das Caput epididymidis gar nicht verdickt. Über die Konsistenz des geschwollenen und verdickten Nebenhodens möchte ich bei dem gehärteten und fixierten Präparate nur mit Vorbehalt ein Urteil abgeben. Er fühlt sich sehr derb an. Der Ductus deferens läßt sich als bleistiftdicker derber Strang durch das umgebende Gewebe hindurch palpieren. Die beiden Blätter der Tunica vaginalis propria sind einen halben Zentimeter voneinander durch eine trübweiße, opake, gallertige Masse getrennt. Auf dem Durchschnitt sieht man statt des feinmaschigen Netzwerkes des Nebenhodens eine gleichmäßig gelblichweiße, von einigen helleren Streifèn durchzogene Schnittfläche.

Zur mikroskopischen Untersuchung wurden Schnitte gefärbt mit Hämatoxylin-Eosin, Methylenblau, Pyronin-Methylgrün, Weigerts Elastinfärbung, nach Van Gieson, Pappenheim und Gram.

Bei der mikroskopischen Untersuchung sieht man die Entzündungsvorgänge in den verschiedenen Teilen des Nebenhodens verschieden heftig auftreten. Das Caput epididymidis ist gänzlich frei von pathologischen Wucherungen, während hingegen das Corpus mäßig starke und die Cauda die stärksten entzündlichsten Veränderungen aufweisen. Aber auch hier trifft man weniger affizierte Kanalschlingen neben stark veränderten an, daneben natürlich auch sämtliche Ủbergänge.

Die Querschnitte der Kanälchen liegen als runde und ovale Lichtungen in dem umgebenden Stroma. Die Lumina zeigen im Vergleich zu normalen VerhäItnissen eine mäßige Erweiterung und sind ausgekleidet von dem Epithel; dieses zeigt noch die Cylinderform und ist pallisadenförmig angeordnet. An der Basis sind noch deutlich die Basalzellen zu erkennen. Nur an einzelnen Stellen des Epithelringes ist der Verlust 
des Flimmerbesatzes zu beobachten. Das Lumen der Kanälchen ist z. T. völlig frei von zelligen Bestandteilen, z. T. enthält es noch einige Spermatozoen.

Daneben liegen Kanälchen, die schon ein weiter fortgeschrittenes Stadium der Veränderung aufweisen. Die Lumina sind nur wenig mehr erweitert. Das Epithel ist völlig flimmerlos geworden, die Cylinderepithelien sind zum größten Teil abgeflacht und haben fast eine kubische Form angenommen. Thre einschichtige Anordnung ist aber noch vollständig erhàlten. Hier treten die ersten Anzeichen einer beginnenden Infiltration auf. Zwischen den Epithelien, aber auch im Lumen der Kanälchen sieht man einige polynucleäre Leukocyten liegen. Das umgebende Bindegewebe ist noch vollständig erhalten, zeigt keinerlei Auflockerung. Die einzelnen Muskelfasern liegen dicht nebeneinander und die elastischen Fasern umgeben als ein feines dichtes Geflecht das Epithel. Überall aber sieht man zwischen den Gewebszügen einzelne polynucleäre Leukocyten meist in der Umgebung von prall gefïllten Capillaren liegen.

Alle diese Veränderungen treten überwiegend im Corpus des Nebenhodens auf. In der Cauda dagegen machen diese mäßigen Veränderungen der Kanälchen schwereren Formen des Entzündungsprozesses Platz, welche hier in überwiegender Zahl das Bild beherrschen.

Am Epithel kann man zunächst alterativ-degenerative Prozesse beobachten. Das Epithel ist nur an noch wenigen Stellen des Kanälchens von cylindrischer Form; in der Hauptsache hat es kubische Form angenommen. Die Zellgrenzen sind verschwommen und undeutlich. Fettige Substanzen sind, allerdings in nur ganz geringer Menge, im Protoplasma der Epithelzellen nachzuweisen. Die Kerne sind mitunter nur ganz schwach und blaß gefärbt erkennbar. Die Zellen selbst sind aufgequollen und zeigen vereinzelt kleinere und größere Hohlräume, Vacuolen (Abb. 1). Das Epithel hat die regelmäßige, pallisadenförmige Aneinanderreihung verloren und liegt unregelmäßig nebeneinander, so daß auch zwischen den Zellen Hohlräume entstanden sind. An manchen Kanälchen kanin man am Epithel proliferative Prozesse neben den alterativen beobachten. Die Zellen liegen nicht mehr in einer Schicht, sondern in mehreren Schichten übereinander; sie sind von regelmäßiger kubischer Form; es ist also ein ausgesprochenes Übergangsepithel geworden.

Auch in dem das Kanälchen umgebenden Gewebe sind die entzündlichen Veränderungen stärker geworden. An der Muskel- und Bindegewebsschicht sieht man eine beginnende Auflockerung. Die einzelnen Fasern werden auseinandergetrieben. Die elastische Faserschicht zeigt in den inneren Teilen noch die normale feinmaschige Struktur, nur an den Randpartien die Anfänge der Auflockerung und Zerklüftung der 
Fasern. Die entzündliche Infiltration hat ebenfalls an Ausdehnung zugenommen. Zu den polynucleären Leukocyten, die an Zahl sehr zugenommen haben, sind vereinzelte eosinophile Leukocyten, Lymphocyten und Plasmazellen gekommen. In reichlicher Menge sind diese Zellelemente zwischen das Epithel und die umgebenden Hüllea einer-

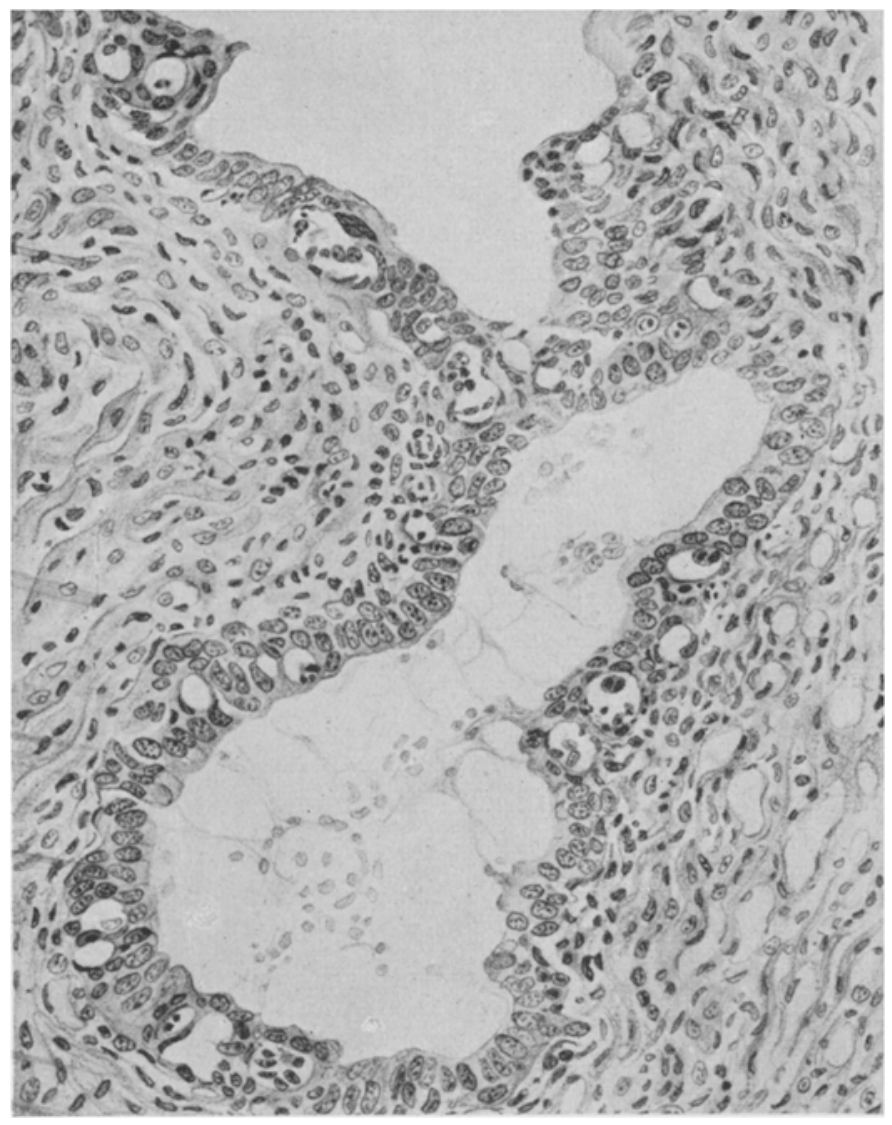

Abb. 1. Vakuolenbildung des Epithels. Beginnende entzündliche Infiltration. Fibrinfäden und polynukleäre Leukocyten im Kanallumen.

seits, die Fasern des Bindegewebes und der Muscularis andererseits eingedrungen and haben deren Auflockerung verursacht. Im Zwischengewebe sieht man die Infiltration peripherwärts längs der Gefäßchen fortschreiten. Aber auch nach dem Lumen des Kanälchen zu haben sich die exsudativen Prozesse fortgesetzt. Im Epithelkranz, sowohl zwischen den einzelnen Zellen als auch in den Epithelien, besonders in den Vacuolen liegen polynucleäre Leukocyten. Bis in das Kanallumen 
sind die Leukocyten vorgedrungen; dieses ist hier angefüllt mit einem Exsudat, das aus geronnenem Fibrin, als feine schwach gefärbte Fädchen zu erkennen, polynucleären, eosinophilen Leukocyten und vereinzelten Plasmazellen besteht. Dazu kommen noch vereinzelte abgestoßene Epithelien, die als Schüppchen mit schlecht gefärbtem Kern oder ohne Kern zwischen den anderen Zellelementen und dem Fibrinnetz liegen.

Andere Kanälchen zeigen wieder ein anderes Bild, ein Bild stärkerer Entzündungserscheinungen. Die das Kanälchen umgebenden Gewebsschichten zeigen einen hohen Grad von Auflockerung; die einzelnen Muskel- und Bindegewebsfibrillen und die Fasern der Elastica - bei letzterer allerdings ausgesprochener in der Peripherie - sind aus ihrem lockeren Gefüge gelöst; z. T. sind sie zugrunde gegangen, z. T. liegen sie verstreut in einem neugebildeten Granulationsgewebe, das zwischen Epithel und Elastica sich besonders kräftig entwickelt hat. Es sind also zum Wiederersatz zerstörter Teile Neubildungsvorgänge eingetreten. In dieser entzündlichen Gewebsneubildung sieht man neben den polynucleären, eosinophilen Leukocyten und Lymphocyten massenhaft Zellen auftreten, die sich besonders hervorheben durch ihr reichliches Protoplasma, den exzentrisch liegenden, radspeichenförmigen Kern und den helleren Protoplasmahof um den Kern. Mit Pyronin-Methylgrün färbt sich das Protoplasma rot. Es sind Plasmazellen, die in dichten Mengen nach der Peripherie zu abnehmend, um das Kanälchen herumliegen. Außerdem liegen verstreut in dem Granulationsgewebe neutrophil und basophil gekörnte Leukocyten und ganz vereinzelte Fibroblasten. Junge Gefäßsprossen dringen in das Granulationsgewebe ein. Zugleich ist auch mit dem Epithel eine weitere Veränderung vor sich gegangen. Das schon mehrreihige Epithel hat an Dicke zugenommen unter gleichzeitiger Umwandlung des Zellcharakters. Es ist mehrschichtig geworden; an der Basis sind die Zellen kubisch, sie zeigen eine mehr oder weniger regelmäßige vieleckige Gestalt. Die Kerne sind gut gefärbt. Diese Zellen liegen nicht mehr dicht aneinander, sondern zeigen feine Protoplasmafortsätze, die z. T. in die benachbarten Zellen ïbergehen. Es sind Stachelzellen mit Intercellularbrücken geworden, so daß man von einem regelmäßigen Plattenepithel sprechen kann, zumal sie nach dem Lumen des Kanälchens zu immer flacher werden, so daß sie an der Oberfläche wie flache Schüppchen liegen. Die Färbbarkeit der Kerne ist nach dem Lumen zu immer undeutlicher geworden. An den obersten Zellen ist ein Kern nicht mehr erkennbar. Diese obersten Epithelzellen, die sich mit Easin blaßrosa färben, werden schließlich in das Kanallumen abgestoßen. Man findet sie dann vereinzelt in dem das Lumen jetzt völlig ausfüllenden Exsudat. Diese mit Eosin sich blaßrosa färbenden platten Epithelschüppchen möchte ich als verhornte Epithelzellen ansprechen, zumal sie sich nach Ernst mit der Gra m schen 
Färbung ausgesprochen blau färben. An einzelnen Stellen beobachtet man noch andersartige proliferative Prozesse am Epithel. Von dem schon an sich verdickten Epithel wachsen Epithelzapfen z. T. von erheblicher Länge in das Granulationsgewebe hinein. An anderen Kanäl-

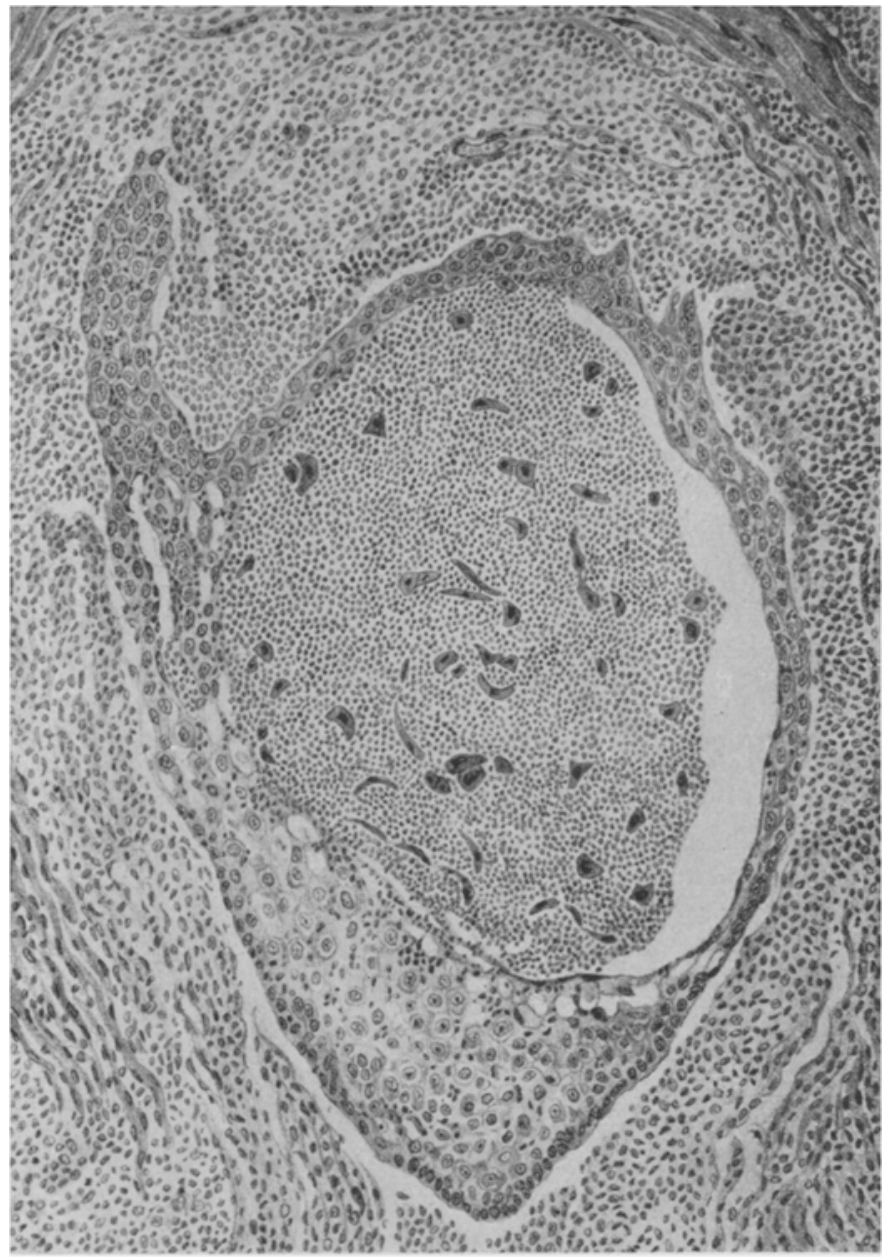

Abb. 2. Plattenepithelbildung. Luksoben ein in das Granulationsgewebe hineinwuchernder Epithelzapfen. Im Exsudat im Kanallumen abgestossene verhernte Epithelschüppchen.

chen wiederum hat das Granulationsgewebe die Oberhand gewonnen. Es hat die Epithellage aufgelockert, sich zwischen die Zellen geschoben und schließlich, die Kontinuität der Zellage gebrochen und gänzlich zerstört. Dann bricht es ein in das Lumen des Kanälchens, um sich mit 
dem darinliegenden Exsudat zu vereinigen, wodurch der völlige Verschluß des Kanälchens bedingt wird (Abb. 2 und 3).

Die verschiedenen Phasen der Umwandlung des Epithels und des Einbruches des Granulationsgewebes in das Kanallumen kann man z. T. an dicht nebeneinanderliegenden, z. T. sogar in denselben Kanälchen beobachten. Z. B. ist in demselben Kanälchen auf der einen Seite noch ein ein- oder zweireihiges kubisches Epithel, auf der anderen Seite schon ein vielschichtiges Plattenepithel mit in das Granulationsgewebe hineingewucherten Zapfen.

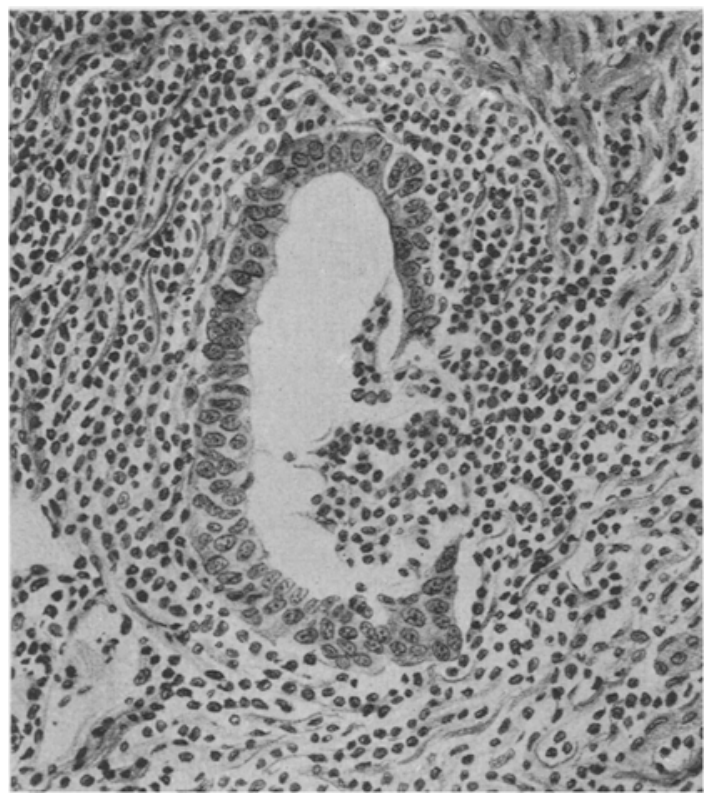

Abb. 3. Einbruch des Granulationsgewebes in das Kanallumen nach Zerstörung des Epithels.

Alle diese alterativen und proliferativen entzündlichen Vorgänge können noch zunehmen und ergeben dann ein Bild vollkommenster Zerstörung und Veränderung der Nebenhodenkanälchen. In dem interstitiellen Gewebe ist es zu einer starken Infiltration zumeist aus Rundzellen längs der Gefäß- und Lymphbahnen gekommen. Das pericanaliculäre Gewebe ist völlig verschwunden. Das Bindegewebe, die Muskulatur und auch die elastische Faserschicht sind untergegangen und durch das wuchernde Granulationsgewebe ersetżt. Das Epithel liegt als lockerer, vielfach durchbrochener Kranz im Granulationsgewebe, in dem auch einzelne abgesprengte Epithelzellen zu finden sind. Diese Epithelreste sind das einzige, das vom Nebenhodenkanälchen übriggeblieben ist. 
Wo das Gefüge des Epithels noch erhalten ist, da findet man das Kanälchen vollständig ausgefüllt mit Exsudat, bestehend aus Fibrin, polynucleären Leukocyten, Lymphocyten, eosinophilen Zellen und abgestoßenen Epithelien, so daß auch hier eine völlige Verlegung des Lumens zustande gekommen ist. Eine Einschmelzung oder Absceßbildung habe ich weder im Nebenhodenkanal noch im Zwischengewebe beobachtet. Aber auch in Granulationsgewebe ist es zu weiteren Veränderungen gekommen. Die neugebildeten Capillarsprossen haben an Zahl zugenommen, desgleichen die Fibroblasten, baso- und neurophilen Leukocyten. Am auffallendsten aber ist die erhebliche Vermehrung der Plasmazellen; sie beherrschen hier vollständig das mikroskopische Bild. Es ist selbstverständlich, daß bis zu dieser letzten ausgedehnten Zerstörung alle möglichen thbergangsstadien zu beobachten sind.

Am Ductus deferens habe ich alle dieselben entzündlichen Veränderungen beobachtet, wie ich sie am Nebenhodenkanälchen zuletzt geschildert habe. Das Granulationsgewebe hat sich hier in der Hauptsache zwischen das Epithel und das umgebende Gewebe hineinentwickelt und das Epithel weit in das Lumen hineingeschoben. Es konnte sich nicht auf Kosten des umgebenden Gewebes entwickeln, wie am Nebenhoden, da sich die drei Muskelschichten und das elastische Fasergeflecht hier als zu kräftig und zu widerstandsfähig zeigten. Trotzdem hat eine starke Infiltration des umgebenden Gewebes mit Auflockerung sowohl der Muskel- und Bindegewebs-, als auch der elastischen Fasern stattgefunden.

Das Cylinderepithel ist zu einem dichten Plattenepithel umgewandelt, das aber auch schon teilweise beginnende Zerstörung aufweist. Das mit Exsudatmassen ausgefüllte Lumen ist bis auf einen schmalen Spalt zusammengedrückt, teils durch die Epithelwucherung, teils durch den Druck der Granulationsgewebe von außen. Dieses hat sich stellenweise in die schon beim normalen Ductus deferens vorhandenen Schleimhautbuchten hineingeschoben und hat diese Falten weit ausgebuchtet und füllt sie mit seinen Zellbestandteilen und Capillarsprossen aus. Auffallend ist hier, daß im Granulationsgewebe die Zahl der eosinophilen Leukocyten beträchtlich zugenommen hat; Plasmazellen überwiegen auch hier.

Gonolokiken habe ich trotz genauesten Nachsuchens in den Präparaten nicht nachweisen können.

Wenn wir nun die Ergebnisse der Untersuchungen zusammenfassen, so hat man es mit einer über den ganzen Kanal des Nebenhodens verbreiteten Entzündung zu tan, die in den verschiedenen Abschnitten des Kanals versehiedene Intensität sowohl der entzündlichen Infiltration als auch der Gewebsschädigung und Zerstörung aufweist. Die stärksten Veränderungen treten in der Cauda, geringere im Corpus epididymidis auf. Die Coni vasculosi im Caput sind frei von entzündlichen Veränderungen. 
Während in den einen Kanalquerschnitten lediglich alterativ-degenerative Veränderungen besonders des Epithels zu finden sind, die in Kernlosigkeit, Verlust der Flimmerhaare, hydropischer Quellung, vacuolärer Degeneration, Abplattung der einzelnen Zellen bestehen, die wiederum als Druckatrophie infolge des in den erweiterten Kanälchen gestauten Sekretes zu deuten ist, ist es in den anderen weitaus meisten Kanalquerschnitten zu einer exsudativen und proliferativen Entzündung gekommen.

Die exsudativen Veränderungen sind in allen Schichten des Kanälehens zu beobachten. Im Lumen wird das Sekret ersetzt dureh ein regelrechtes Exsudat mit Fibrin, polynucleären Leukocyten, Lymphocyten und Plasmazellen. Das degenerierte Epithel wird durchsetzt von Exsudatzellen, es wird aufgelockert und z. T. ins Lumen abgestoßen. Auch in den Wandschichten tritt die exsudative Form auf, die an Intensität immer mehr zunimmt; die Wandschichten werden aufgelockert; sowohl die Muskel- und Bindegewebsschicht als auch die Elastica zeigt eine Auflockerung der einzelnen Fasern bei gleichzeitiger Einwanderung von Exsudatzellen. Im interstitiellen Gewebe schreitet die entzündliche Infiltration längs der Gefäße und Lymphbahnen weiter. Zu gleicher Zeit sind die degenerativen Prozesse noch weiter vorgeschritten. Das Bindegewebe, die Muskelschicht und auch die Elastica gehen bei den stärker entzündlich affizierten Kanälchen allmählich zugrunde bis zum völligen Schwunde.

Während diese alterativen und exsudativen Formen der Entzündung hauptsächlich im. Corpus des Nebenhodens zu beobachten sind, überwiegen in der Cauda die proliferativ-reparativen Prozesse. Sie bestehen in der Wucherung, dem Mehrschichtigwerden und der Veränderung des Zellcharakters des Epithels und der Bildung eines Granulationsgewebes. Die Plasmazellen haben so an Zahl zugenommen, daß sie das Bild völlig beherrschen. Auch die eosinophilen Zellen, die neutround basophilen Leukocyten haben sich vermehrt; schließlich sieht man überall im Granulationsgewebe Fibroblasten liegen und zahlreiche Capillarsprossen sich entwickeln. Das Granulationsgewebe hat sich zunächst zwischen Epithel und der Elastica entwickelt, sich dann nach der Zerstörung der Elastica weiter peripherwärts ausgebreitet und ist nach Durchbrechung der Epithellage ins Kanallumen hineingewuchert, so daß es zum Verschluß und Untergang des ganzen Kanälchens gekommen ist.

Obgleich ich nicht bei meinen Untersuchungen Gonokokken nachweisen konnte, bin ich doch der Überzeugung, daß es sich in dem beschriebenen Falle mit größter Wahrscheinlichkeit um eine Epididymitis gonorrhoica handelt. Denn unter den Erkrankungen des Nekenhoder s habe ich in der mir zugänglichen Literatur dieselben oder ähnliche. 
pathologisch-histoiogische Veränderungen nux bei cier Gonorthos des Nebenhodens gefunden. Weiterhin spricht für eine Gonorrhoe der vor und nach der Operation bestehende Ausfluß aus der Harnröhre, wenn auch eine Infectio ven. geleugnet und eine bakterioskopische Untersuchung unterlassen wurde. Auch würde der negative Gonokokkenbefund eine gonorrhoische Erkrankung des Nebenhodens nicht ausschließen, denn in älteren gonorrhoischen Prozessen sind Gonokokken nicht oder sehr schwer nachzuweisen. Ob die Entzündung durch die Gonokokken selbst oder durch deren Toxine hervorgerufen worden ist, wage ich nicht zu entscheiden. Gestützt wird meine Annahme durch die Arbeiten von Nobl und Sellei, die dieselben Veränderungen beschrieben haben, ohne Gonokokken nachweisen zu können. In meinem Falle kann ich die Beobachtung Nobls usw, daß die Erkrankung besonders die Cauda, weniger das Corpus und gar nicht das Caput epididymidis befällt, vollkommen bestätigen.

Bei meinen Untersuchungen und Ergebnissen möchte ich besonders hinweisen auf einige Veränderungen, die bisher noch nicht genügend berücksichtigt worden sind. Das sind erstens die Veränderungen an der elastischen Faserschicht. Baermann fand die Elastica bei seinen Fällen nur wenig alteriert; ich dagegen konnte die Zerstörung von der Auffaserung bis zum völligen Zugrundegehen beobachten. Daß3 sie am längsten von den umgebenden Schichten den entzündlichen Schädigungen Stand hält, geht aus meinen Beschreibungen hervor. Dann habe ich die Entwicklung eines regelrechten Granulationsgewebes beobachten können, die zunächst zwischen Epithelschichten und umgebendem Gewebe auftrat, dann sich ausbreitete sowohl peripherwärts als auch nach dem Lumen zu. Man kann hier Parallelen ziehen zwischen Epididymitis gonorrhoica und Salpingitis gonorrhoica. Man kann geradezu von spezifischen Bildern auf dem Höhepunkt der Erkrankung sprechen. Wie bei der Salpingitis gonorrhoica beginnt die plasmacelluläre Infiltration subepithelial, zeigt das Epithel reichliche Durchwanderung von Leukocyten und Plasmazellen. Vor allem scheint das Auftreten von so zahlreichen Plasmazellen vorwiegend bei gonorrhoischen Erkrankungen der Genitalorgane vorzukommen (Schridde), wenn solche auch, wie spätere Untersuchungen gezeigt haben, bei nicht gonorrhoischen Erkrankungen mitunter beobachtet worden sind. Für die sowohl bei der gonorrhoischen Salpingitis, als auch in meinem Falle beobachtete Zerstörung des Epithels findet man Analogien bei der Bronchiolitis obliterans und bei der Mastitis obliterans. Auch bei der Bronchiolitis obliterans ist das Lumen der betreffenden Bronchiolen verengt oder völlig verschlossen durch ein Granulationsgewebe. Entweder ist es durch Organisation des liegen gebliebenen Exsudates oder durch Einbruch des Granulationsgewebes von außen zur Obliteration gekommen. Der oft noch erhal- 
tene Lumenspalt ist dann mit gewuchertem Epithel ausgefüllt. Bei der obliterierenden Mastitis geht mit zunehmender Proliferation des Granulationsgewebes eine Verengerung des Lumens einher. Hier findet allerdings eine völlige Destruktion des auskleidenden Epithels statt, die ich in meinem Falle von gonorrhoischer Epididymitis nicht beobachten konnte. Vielmehr kommt es hier aufer durch die Proliferation des Granulationsgewebes mit evtl. Einbruch in den Kanal durch eine hochgradige Wucherung des Epithels zur Obliteration des Lumens. Diese Wucherung des Epithels mit gleichzeitiger Umwandlung des Zellcharakters vom Cylinderepithel zum regelrechten Plattenepithel ist die dritte Veränderung, die m. E. bis jetzt bei der Epididymitis gonorrhoica noch nicht beschrieben worden ist. Es wurde wohl eine Umwandlung des Cylinderepithels in Plattenepithel beobachtet, aber das Auftreten von Verhornung, also Epidermisierung des Plattenepithels, und welche Art von Zellumwandlung vorliegt, habe ich nirgends in der Literatur finden können. Diese Zellumwandlung mit Verhornung ist am männlichen Genitalapparat bei Gonorrhoe schon beobachtet worden. So hat 1887 Neelsen sie beschrieben bei 14 Fällen von chronischer gonorrhoischer Urethritis. Stets war, wo auf der chronisch entzündeten Schleimhaut Plattenepithelbildung auftrat, eine Verhornung der obersten Schichten vorhanden. In den von Cedercreutz beschriebenen Fällen kommt es bei Urethritis anterior ebenfalls zur Bildung von verhornendem Plattenepithel. Er hält es für wahrscheinlich, daß die durch Gonorrhoen hervorgerufenen Epithelveränderungen sich immer aus von der Embryonalzeit her persistierenden Plattenepithelinseln entwickeln, während Neelsen eine echte Metaplasie im Sinne Virchows angenommen hat. Analoge Fälle von dieser Zellumwandlung sind in der pathologisch-anatomischen Literatur an verschiedensten Organen bei Entzündungen nachzuweisen. Neuerdings hat sie Askanazy beobachten können in der Trachea bei der Influienza. Er unterscheidet neben einfacher Kongestion und einfachem Katarrh der Luftwege noch den mit Pflasterepithelbildung einhergehenden Katarrh. Eine epidermale Umwandlung, d.h. Erscheinungen von Verhornung hat er nicht feststellen können.

$\mathrm{Da} ß$ in unserem Falle eine ausgesprochene Zellumwandlung vorliegt, ist sicher, sie ist bedingt durch die veränderten Lebensbedingungen der Zellen; in unserem Falle durch die Toxine der Gonokokken, durch die Entzündung und durch den Druck in den Kanälchen, also durch funktionelle und chemische Beanspruchung.

Welche Art von Zellumwandlung liegt nun in unserem Falle vor? Eine Heteroplasie nach Schridde, wie sie in der Arbeit von Cedercre utz anzunehmen ist, kann man wohl ausschließen. Denn ich halte es nicht für wahrscheinlich, daß die Zellumwandlung von Zellen aus- 
geht, die indifferent geblieben sind, sondern es handelt sich lediglich um eine Zellumwandlung nach abgeschlossener Körperdifferenzierung. Ich nehme also keine Störung im Laufe der ontogenetischen Entwicklung an; denn die abführenden Samenwege, der Ductus deferens und Ductus epididymidis werden aus der Urniere gebildet und diese ist mit Cylinderepithel ausgekleidet. Die von R. Me yer beschriebenen Hornepithelgebilde, die er den suprasexualen Kanälchen des Wolff schen Körpers zurechnet, kommen als Ausgangspunkt nicht in Betracht, da er sie stets deutlich gettrennt von Epididymiskanälchen gefunden hat. Würde man sie berücksichtigen, so müßte man nach Schridde eine Prosoplasie annehmen, eine Ansicht, der R. Me yer nicht beistimmt. Wenn man also die Hornepithelgebilde Meyers als Ausgangspunkte der Zellumwandlung nicht berücksichtigk, muß man zu dem Schluß kommen, daß in unserem Falle von gonorrhoischer Epididymitis eine indirekte Metaplasie nach Schridde vorliegt. . Es ist nach Zerstörung der Cylinderepithelien eine Regeneration eingetreten, aber nicht wieder zu dem ursprünglichen Cylinderepithel, sondern mit Umdifferenzierung in ein regelrechtes Plattenepithel. Noch genauer ausgedrückt handelt es sich um eine anaplastische Metaplasie oder' nach Ribbert um Rückschlag, da eine Umwandlung von höher in geringer differenzierte Zellen stattgefunden hat.

Wie Askanazy bei der Influenza die Epithelmetaplasie als Schutzmittel betrachtet, könnten auch wir bei der Epididymitis gonorrhoica annehmen, daß die durch Metaplasie entstandene dichtere und festere Plattenepitheldecke den Gonokokken und deren Toxinen den Eintritt in die Gewebe erschwert und ihre Wachstumsbedingungen auf dem Plattenepithel geringere sind als auf dem normalen Cylinderepithel.

\section{Literaturverzeichnis.}

1) Aschoff, Pathologische Anatomie, Lehrbuch. Jena 1911. - 2) Askanazy, Über die Veränderungen der großen Luftwege, besonders ihre EpithelMetaplasie bei der Influenza. Korrespondenzblatt f. Schweiz. Ärzte 1919, Nr. 15. - $\left.{ }^{3}\right)$ Andr y und Dalous, Lésions histologiques de l'epididymite blennorrhagique. Ann. de dermatol. et de syphil. 1903. Ref. Centralbl. f. allg. Pathol. 1903. 4) Baermann, Über die Pathogenese der gonorrhoischen Epididymitis und über Versuche, dieselbe durch Punktion zu behandeln. Dtsch. med. Wochenschr. 1903, Nr. 40. $-{ }^{5}$ ) Baermann, Weiterer Beitrag zur Pathologie der gonorrhoischen Epididymitis.' Arch. f. Dermatol. u. Syphilis 78, 1905. - 6) Baer mann, Über die Pathogenese der gonorrhoischen Epididymitis. VIII. Kongreß der deutschen dermatologischen Gesellschaft, Sarajevo 1903. - 7) Bonner, Akute Epididymo-Orchitis verursacht durch Bacillus coli. The Lancet 1913. Ref. Arch. f. Dermatol. u. Syphilis. 119, 1915. - ${ }^{8}$ ) Bruck, Pathologie der Gonorrhoe. Ergebnisse der allgemeinen Pathologie 1912. - ${ }^{9}$ ) Cedercreutz, Axel, Zur Kenntnis der Topographie des Plattenepithels der männlichen Urethra im normalen und patholog. Zustande. Archiv f. Dermatol. u. Syphilis 79, 19/06. '10) Delbet, Anastomose zwischen Nebenhoden und Vas deferens. Gazette Méicale 
de Paris 1912, Nr. 134. Ref. Arch. f. Dermatol. u. Syphilis 115, 1913. - $\left.{ }^{11}\right)$ Delbet und Chevassu, Les oblitérations blennorrh. de l'epididym. Ann. malad. gén. ur. 1908, Nr. 16/17, zit. bei Bruck $\left({ }^{8}\right) .-{ }^{12}$ ) Dind und Métra $u x$, Sur les accidents testiculaires de l'origine blennorb. Ann. de ther. derm. 1908, Nr. 1l, zit. bei Br uck. - $^{13}$ ) Gro B, Deferentitis und Epididymitis gonorrhoica. Handbuch der Geschlechtskrankheiten 1912. - ${ }^{14}$ ) Ingier, Über obliterierende Mastitis. Virchows Archiv 198, 1509. - 15) Jores, Pathologie der Harn- und männlichen Geschlechtsorgane. Ergebnisse der allgemeinen Pathologie 1907. - 16) Jordan, Ein Beitrag zur Statistik der Epididymitis gonorrhoica. Arch. f. Dermatol. u. Syphilis $\boldsymbol{\gamma}_{2}, 1904$. 17) Ka ufma nn, Lehrbuch der speziellen patho-logischen Anatomie. Berlin 1907. $\left.-{ }^{18}\right) \mathrm{Keibel}$, Handbuch der Entwicklungsgeschichte des Menschen. Leipzig 1911. - ${ }^{19}$ ) Kölliker, Handbuch der Gewebelehre des Menschen. Leipzig 1902. - ${ }^{20}$ ) Königsfeld und Salzmann, Der Diplococcus crassus als Erreger von Urethritis und Epididymitis. Arch. f. Dermatol. u. Syphilis 120, 1914. 21) Meyer, Robert, Über embryonale Gewebsanomalien und ihre pathologische Bedeutung im allgemeinen und solche des männlichen Genitalapparates im besonderen. Ergebn. d. allg. Pathọl. 1911. - ${ }^{22}$ ) Müller, Epididymitis und Bacterium coli commune. Münch. med. Wochẹnschr. 1909, Nr. 50. - ${ }^{23}$ ) Neels e n, Über einige histologische Veränderungen in der chronisch entzündeten männlichen Urethra. Arch. f. Dermatol. u. Syphilis 19, 1887. - ${ }^{24}$ ) Neisser und Schä fer, Gonokokken. Ergebnisse der allgemeinen Pathologie 1896. - ${ }^{25}$ ) Nobl, Zur Histologie der blennorrhoischen Deferentitis und Epididymitis. Arch. f. Dermatol. u. Syphilis 67, 1903. - ${ }^{26}$ ) Nobi, Über postblennorrhoische Wegsamkeit des Nebenhodens. VerhandI. d. 78. Versamml. Deutscher Naturforscher und Ärzte, Stuttgart 1906. - ${ }^{27}$ ) Op pen heim und Löw, Klinische und experimentelle Studien zur Pathogenese der gonorrhoischen Epididymitis. Virchows Archiv 182, 1905. $-{ }^{28}$ ) Reynolds, Epididymitis verursacht durch Bacillus coli. Amer. journ. of the med. sciences 1913. Ref. Arch. f. Dermatol, u. Syphilis 119, 1915. 29) Routier, Orchite blennorrhagique suppurée présence du gonocoque dans le pus. Méd. mod. Juillet 1895. Ref. Arch. f. Dermatol. u. Syphilis 38, 1897. $\left.{ }^{30}\right)$ Schäffer, Gonokokken. Ergebnisse der allgemeinen Pathologie 1897. - 31) Schäffer, Pathologie der Gonorrhoe. Ergebnisse der allgemeinen Pathologie 1902. - ${ }^{32}$ ) Schindler, Über antiperistaltische Bewegung des Vas deferens und die Behandlung der akuten gonorrhoischen Urethritis posterior. Arch. f. Dermatol. u. Syphilis 85, 1907. - ${ }^{33}$ ) Schridde, Die ortsfremden Epithelgewebe des Menschen. Jena 1909. - ${ }^{34}$ ) Sellei, Beiträge zur Histologie der Epididymitis gonorrhoica. Dermatol. Zeitschr. 11, 1904. - 35 ) Si m monds, Ursache der Azoospermie. Dtsch. Arch. f. klin. Med. 61, 1898. - ${ }^{36}$ ) Si m monds, Über narbigen Verschluß des Samenleiters. Verhandlungen der deutschen Pathologischen Gesellschaft, I. Tagung 1899. - ${ }^{37}$ ) De Sinéty, Bericht der Société de biologie in Paris 1895. Ref. Ziegler, Centralbı. f. allg. Pathol. u. pathol. Anat. 1896. - ${ }^{38}$ ) Stöhr, Lehrbuch der Histologie. Jena 1912. - ${ }^{39}$ ) Sprecher, Beitrag zum Studium der gonorrhoischen Epididymitis. Riforma med. Nr. 20. Ref. Ziegler, Centralbl. f. allg. Pathol. u. pathol. Anat. 1905. - ${ }^{40}$ ) v. Winiwarter, Die Erkrankungen des Penis, Hodens und der Hüllen des Hociens. Handbuch der Urologie 1906. - $\left.{ }^{41}\right)$ Wi t.te, Zur Pathogenese der gonorrhoischen Epididymitis. Arch. f. Dermatol. u. Syphilis 50, 1899. $-{ }^{42}$ ) Wossidlo, Die Gonorrhoe des Mannes. Berlin 1903. ${ }^{43}$ ) v. Zeiß1, Die venerischen Erkrarkungen der Harnröhre. Handbuci der Urologie 1906. 